\title{
A Dynamic Semantic Account of the Temporal Interpretation of Noun Phrases
}

\author{
Judith Tonhauser \\ Stanford University
}

\section{Introduction}

In general, the temporal interpretation of natural language utterances is concerned with identifying the temporal location of properties and relations expressed within an utterance with respect to contextually salient time points, e.g., the time of utterance. Most of the literature on temporal interpretation has been concerned with the temporal interpretation of verbal predicates and their verbal projections. In (1), the property 'sleep' expressed by the verbal predicate slept is temporally interpreted at some time prior to the utterance time, as indicated by past tense morphology.

(1) Every student slept in the library.

In this paper I am concerned with the temporal interpretation of nominal predicates and their projections; the paper is restricted to NPs denoting individuals. In (1), the nominal predicate student contained within the NP every student expresses the property 'student' which, similarly to the property 'sleep', is true of the individuals denoted by the NP at a particular time. However, unlike verbal predicates, nominal predicates in languages like English and German are not marked by tense morphology which would indicate the time at which the property 'student' is true of the individuals. In (1), we understand the property 'student' to be true of the individuals at the time at which the verbal predicate is interpreted, i.e., the individuals were students when they slept in the library. But how is this interpretation determined? In her pioneering work on the temporal interpretation of NPs, Enç (1981) demonstrates that nominal predicates are not necessarily interpreted at the time of the verbal predication. In (2), for instance, the property 'hostage' is understood to be true of the individuals denoted by the NP every hostage at a time prior to the time of the verbal predication, which is later than the utterance time as indicated by verbal tense morphology.

John will meet every hostage at the president's party.

Enç (1981) p.38

Enç (1981) proposes to analyze NPs as indexicals in order to liberate their temporal interpretation from the tense operator introduced by verbal tense morphology. The temporal interpretation of an NP is then contextually determined and the analysis accommodates Enç's claim that "[w]hen we use a noun, we seem to be able to talk about ANY set of individuals we please without being restricted by the moment of evaluation of verbs" (Enç (1981:45), emphasis in the original). The analysis I develop here is in the spirit of Enç's to the extent that the temporal interpretation of an NP is contextually determined; however, I argue that Enç's claim is too liberal because there are clear constraints on the possible times at which a nominal predicate can be interpreted. In the dynamic semantic account that I develop in this paper the 
temporal interpretation of a nominal predicate embedded within an NP is contextually determined: it is interpreted at the time of the verbal predication unless there is contextual justification for the nominal predicate to be evaluated at an alternative time. The paper is organized as follows.

In section 2 I demonstrate how the context affects the temporal interpretation of nominal predicates and present a formal account of the temporal interpretation of NPs within Discourse Representation Theory (DRT; Kamp (1981), Kamp \& Reyle (1993)). Intuitively, NPs identify the individuals involved in the utterance via the property expressed by the nominal predicate. These individuals may either be identified by a property that is true of these individuals at the verbal predication time, as the most salient time of the utterance, or the individuals can be identified via a property which is salient for these individuals in the context already-and in this case the property expressed by the nominal predicate does not need to be true of the individuals at the verbal predication time. Since different types of NPs (e.g., definite, quantificational, indefinite NPs) interact with the context in distinct ways, I demonstrate that we find different possibilities for the temporal interpretation of a nominal predicate depending on the type of NP it is embedded in. The formal account is extended in section 3 to include temporal $\mathbf{N}^{\prime}$-modifiers which determine the temporal interpretation of the nominal predicate and possibly also interact with that of the verbal predicate. In section 4 I discuss some empirical problems with Musan's $(1995,1999)$ analysis which claims that there exists a type of NP which must obligatorily be interpreted at the verbal predication time. Section 5 presents some cross-linguistic data which further supports the dynamic analysis developed here. Section 6 concludes the paper.

\section{The Temporal Interpretation of Nominal Predicates}

Nominal and verbal predicates are alike in that they express properties which are true of particular individuals at particular times. Thus, the property 'student' is true of me at the time of the writing of this paper but probably won't be true in 10 years, and, similarly, the property 'sleep' is true of me at some times but not at others. These parallels between nominal and verbal predicates and more direct evidence from such phrases as former student, in which the evaluation time of the nominal predicate is modified, motivate the assumption that nominal as well as verbal predicates should have a temporal argument and receive a temporal interpretation. ${ }^{1}$ Thus, parallel to verbal predicates, the semantic representation of a nominal predicate must include a temporal argument which identifies the time at which the property expressed by the nominal predicate is true of the individuals. In the semantic representation of the nominal predicate student of (1), s:student( $\mathrm{x})$, the temporal argument $s$ represents the state during which the property 'student' is true of the individuals $x$, parallel to the representation $s: \operatorname{seep}(x)$ for the verbal predicate of the utterance. While the temporal location of the time at which the verbal predicate is true is determined by tense morphology (and possibly temporal adverbials), no such morphology is available for nominal predicates, at least in languages like German and English. ${ }^{2}$ What, then, determines the time at which a particular nom- 
inal predicate is interpreted? I claim that the answer to this question relates to the function of NPs within utterances, which is to identify the individuals that the finite verbal predicate is predicated of. The finite verbal predicate within any utterance attributes a property to a (set of) individual(s) at a particular time as determined by tense (and possibly temporal adverbs). While nominal predicates, too, attribute a property to individuals, the crucial difference between nominal and verbal predicates in their use within a proposition is that the property expressed by a nominal predicate embedded within an NP serves to identify the individuals denoted by the NP. The property expressed by the nominal predicate must uniquely identify a particular (set of) individual(s)-and since every utterance is situated in time, the property must uniquely identify these individuals at a particular time. Intuitively, there seem to be two ways for a nominal predicate to identify a particular (set of) individual(s), each of which, as we will see, has distinct consequences for the temporal interpretation of the nominal predicate. I informally discuss these two possibilities in turn now. (The next sections provide a formal account.) First, we can use a nominal predicate that expresses a property which is true of the individuals at the verbal predication time. For instance, if a friend asks me how the pool competition went last night, I could utter (3), where the nominal predicates student and retiree each expresses a property which is understood to apply to the individuals at the verbal predication time and thereby allows a particular set of individuals to be identified.

(3) The students did a good job, but the retirees were in much better shape.

Thus, we can identify individuals with a property which is true of the individuals at the verbal predication time. I argue that this is the default temporal interpretation for nominal predicates, because the verbal predication time is the most salient time in an utterance. This default interpretation also accounts for why we understand the example in (1) such that the property student is true of the individuals at the time at which they slept although there is no overt indication for this interpretation of the nominal predicate.

The second possibility is to use a nominal predicate that expresses a property which in the particular context is highly salient for a particular set of individuals. This possibility does not require the property to be true at the verbal predication time. Consider a context for (2) in which we have been made aware of some individuals being taken hostage and the newspapers referred to these individuals as the hostages in discussing their living-conditions and fate over the weeks that they are kept captive. At some point, we learn that these individuals have been set free again and that there is a party to celebrate their return. These individuals can still be uniquely identified with the nominal predicate hostage in John will meet every hostage at the party because this is the property most salient for these individuals and allows us to uniquely identify them although the property is no longer true of the individuals at the time of utterance or at the verbal predication time. Thus, when we denote a particular set of individuals with an overt NP, we use a property which is salient for the individuals in the particular context: this can either be a property which is true of the individuals at the most salient time of the utterance, i.e., the 
verbal predication time, or a property which is salient for the individuals in the context. In the latter case, the temporal interpretation of the nominal predicate is determined by what has already been established for the individuals in the context. This will be demonstrated more explicitly in section 2.2 where I illustrate how the interpretation of different types of NPs (definite, indefinite, quantificational, etc.) in context affects the temporal interpretation of the embedded nominal predicate. Before I turn to these distinctions in section 2.2, I present the formal background.

\subsection{The Temporal Dimension of Nominal Predicates}

The formal account of the temporal interpretation of NPs is developed within DRT. As mentioned above, I assume that nominal predicates, parallel to verbal predicates, introduce a semantic representation that includes a temporal argument $s$ that represents the state during which the property or relation expressed by the predicate is true of the individuals. Thus, the nominal predicate student introduces a representation s:student $(\mathrm{x})$ where $\mathrm{s}$ represents the state during which the property 'student' is true of the individuals $x$. This state $s$ is temporally situated at time $t$ which for finite verbal predicates is bound by tense, but for nominal predicates needs to be contextually resolved. In DRT, it is assumed that natural language expressions have context-dependent as well as context-independent parts of meaning, and this is modeled in the representations assigned to such expressions: each representation consists of a Discourse Representation Structure (DRS) which represents the asserted material as well as a set of (possibly empty) context-dependent meaning parts (van der Sandt (1992)). When computing the representation of a complex natural language expression the asserted and the context-dependent part of the representation of the complex expression are each computed from the asserted and the context-dependent meaning parts of the parts of the complex expression. A final, resolved DRS may not contain context-dependent meaning parts; rather, these must be resolved in the context. The representation of nominal predicates I propose in (4) adopts this partition to model the context-dependency of the temporal interpretation of a nominal predicate. Following van der Sandt (1992) and Kamp (2001a, $2001 \mathrm{~b}$ ), the lexical representation in (4) is a tuple where the first element is a set of context-dependent meaning parts and the second element is a DRS consisting of the asserted meaning of the lexical element.

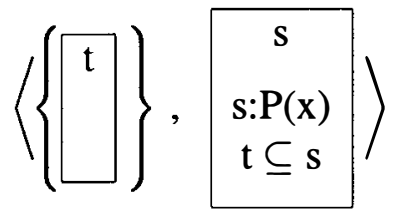

The DRS in the second element of (4) introduces a state discourse referent (DR) $s$ which represents the time at which the property $\mathrm{P}$ expressed by a nominal predicate is true of the individuals $\mathrm{x}$. This state DR $\mathrm{s}$ is existentially bound and, as mentioned above, it is temporally located by a temporal DR $t$; the condition $t \subseteq$ s requires that $s$ lies at time t. The first element of (4) consists of the context-dependent meaning elements of the lexical representation of a nominal predicate and thus indicates 
that the DR $\mathrm{t}$ needs to be contextually resolved. (The DR $\mathrm{x}$ is either bound by a determiner or by the context.)

The representation of nominal predicates in (4), in principle, allows nominal predicates to be interpreted at any available time in the context to which the DR $t$ is resolved. This is basically what Enç (1981) argues for. However, I argue here that a nominal predicate is interpreted at the verbal predication time, unless there exists contextual justification for the nominal predicate to be temporally interpreted at a different time. This is formulated in (5) as a condition on the resolution of the temporal DR $\mathrm{t}$ of nominal predicates.

CONDITION ON THE Resolution of THE TEMPoRAL DR OF A NOMINAL PREDICATE: Unless there exists contextual justification to the contrary, the temporal DR $t$ of a nominal predicate is by default interpreted at the verbal predication time.

Given (5), the temporal interpretation of NPs is rather restricted (in contrast to Enç's account), since only those NPs may be interpreted at a time distinct from the verbal predication time for which there exists contextual justification. Before I discuss the distinct predictions (5) makes for different types of NPs in the next section, I illustrate how (5) accounts for the temporal interpretation of (1). In the unresolved DRS for (1) in (6), the nominal and the verbal predicate introduce state DRs $s$ and $s^{\prime}$, respectively, which are temporally located by DRs $t$ and $t^{\prime}$, respectively. Tense morphology introduces a temporal DR $t_{t}$ (following Reyle, Rossdeutscher and Kamp (2000)), which is located prior to the time of utterance (represented as $n$ ) as indicated by the condition $\mathrm{t}_{t} \prec n$. The $\mathrm{DR} \mathrm{t}_{t}$ binds the temporal DR $\mathrm{t}^{\prime}$ introduced by the verbal predicate. The temporal DR $t$ introduced by the nominal predicate student in accordance with the representation proposed in (4) is not yet resolved, i.e., not yet identified with a temporal DR available in the context. The top line of the DRS in (6) indicates that there are two temporal discourse referents accessible for the resolution of $t$, namely the time of utterance $n$ and the time $t_{t}$. (The DRS is simplified to the extent that I have omitted the contribution of the prepositional phrase in the library, as well as the anaphoric properties of the past tense.)

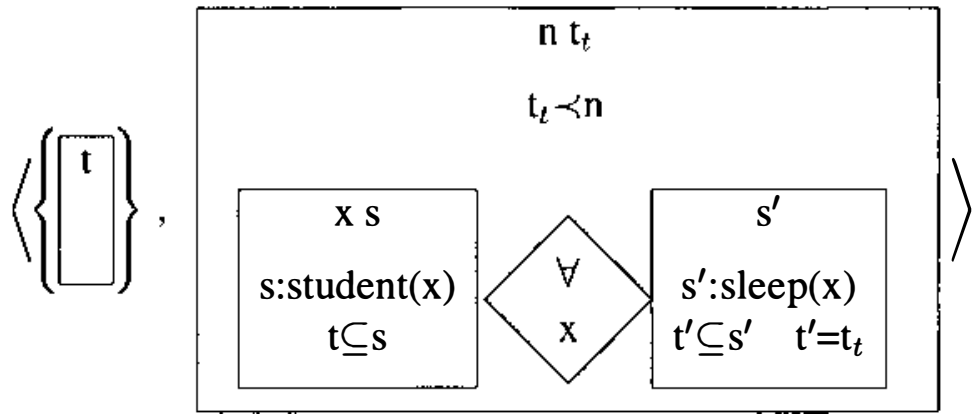

Since there is no contextual justification to the contrary, by (5) the DR $t$ is resolved to $t_{t}$ as indicated in the resolved DRS in (7). This DRS represents the desired interpretation of (1) in which the individuals denoted by the NP every student are students at the time at which they slept in the library. 


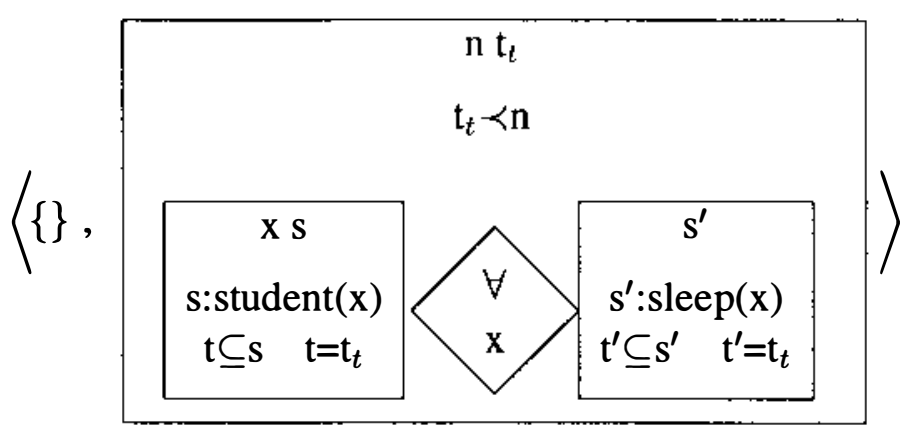

I now demonstrate how the context may affect the temporal interpretation of nominal predicates embedded in different types of NPs.

\subsection{The Temporal Interpretation of Nominal Predicates in Context}

A central feature of dynamic semantics as formulated, for instance, in Kamp \& Reyle (1993) is that the interpretation of NPs is context-dependent. For instance, definite NPs presuppose that their referent is already established in the context and the interpretation of quantificational NPs is contextually restricted. Different types of NPs interact with the context in distinct ways. The dynamic interpretation of NPs has immediate consequences for the temporal interpretation of the embedded nominal predicate since the condition in (5) specifies that there needs to be contextual justification for the nominal predicate not to be interpreted at the verbal predication time. In this section I illustrate how (5) interacts with the dynamic interpretation of NPs: generally, a temporal interpretation of the nominal predicate at a time other than the verbal predication time is more easily justified for NPs whose interpretation is typically thought of depending on individuals or properties established in the context, but I demonstrate that it is in principle possible for all types of NPs.

As mentioned, quantificational NPs like every hostage in (2) are contextually restricted. Recall the context for (2) that I gave above in which we are aware of a set of individuals who have been hostages at some earlier time but who are free now. In (2) we may still uniquely identify these individuals using the property 'hostage'. (8) gives the formal representation of the (partial) context and the unresolved DRS of (2) (where I have again omitted representing elements irrelevant to the discussion). I demonstrate below how the temporal DR $\mathrm{t}$ is resolved.
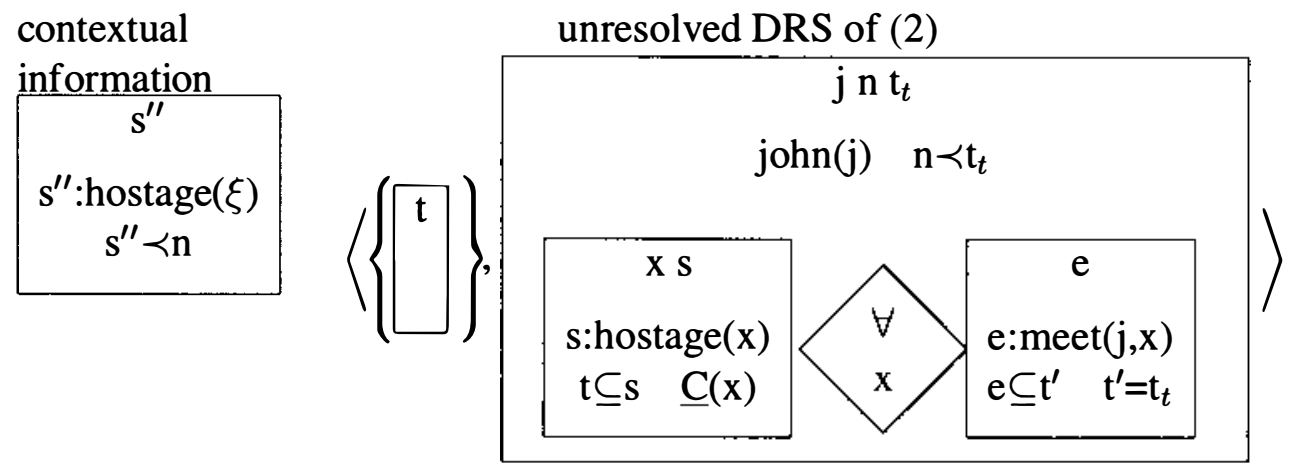

The DRS on the left of (8) represents the (partial) context I assume for (2). It spec- 
ifies a time time $s^{\prime \prime}$ which lies prior to the utterance time $\mathrm{n}$ and during which the property 'hostage' is true of a particular set of individuals $\xi$. This is the context against which the unresolved DRS of (2), given to the right of (8), is interpreted. Following Kamp \& Reyle (1993), the quantifier every is contextually restricted as indicated by the condition $\underline{\mathrm{C}}(\mathrm{x})$. The contextual information in (8) is part of these contextual restrictions $\underline{\mathrm{C}}(\mathrm{x})$ : the resolution of these restrictions includes the identification of the individuals denoted by every hostage with the individuals established in the context, formally accounted for with the condition $x \in \xi$. Once the individuals have been identified, the temporal interpretation of the property 'hostage' in the unresolved DRS must be consistent with what has already been established for the individuals $\xi$ in the context. Thus, $s$ is identified with $\mathrm{s}^{\prime \prime}$ which results in a resolved DRS of (2) in which the individuals denoted by the NP every hostage are hostages at some earlier time $s^{\prime \prime}$ rather than the verbal predication time or the utterance time. Thus, the context I have assumed here for (2) justifies that the nominal predicate is not interpreted at the verbal predication time: the individuals are identified with a contextually established set of individuals and the properties associated with these individuals require the property 'hostage' of (2) to be interpreted at a time preceding the verbal predication time and the utterance time.

The contextual justification for the non-default interpretation of (5) that I demonstrated with (2) in the particular context above relies on the contextual restriction of the interpretation of quantificational NPs. When I first introduced (2) on page 1 it seemed as if the non-default interpretation of the nominal predicate was possible even without a justifying context, for instance, because it might be implausible that hostages attend parties. Thus, it seemed that the lexical semantics of hostage and world knowledge about typical attendees of parties alone would justify the non-default interpretation of (2). This, however, I argue is not the case. Rather, with quantificational NPs, lexical semantic constraints might facilitate the non-default temporal interpretation of the nominal predicate, but contextual justification in the way I demonstrated above is still necessary. This is demonstrated by the context for (2) in (9), which does not give a contextual justification for an interpretation of the nominal predicate of (2) at a time distinct from the verbal predication. In this context, the nominal predicate hostage in (2) is interpreted at the verbal predication time; world knowledge and the lexical semantics of hostage that might have supported a non-default interpretation are overridden.

(9) The hostages are brought into a room (filled with people celebrating the president's birthday) by their captors with guns held to their backs.

Although it is important in general to recognize the lexical semantic contributions of the nominal predicate to its temporal interpretation, contextual justification of the type illustrated above must be available in order for quantificational NPs to be interpreted at a time distinct from the verbal predication time. ${ }^{3}$ (The temporal interpretation of generic bare plurals, discussed below, is more affected by these lexical semantic constraints.)

Definite NPs (headed by the determiner the) presuppose that their referent is 
already established in the context (Kamp \& Reyle (1993)). For instance, the definite NP the man in (10) refers back to the individual introduced in the first sentence by the indefinite NP a man.

(10) A man and a woman entered a bar. The man was wearing a hat.

In the discourse in (11), the requirement that definite NPs refer to a discourse participant already established in the context affects the temporal interpretation of the embedded nominal predicate. A set of individuals is introduced in the context by A; these individuals are contextually specified to be members of the government at the time of utterance. This is the context against which the definite NP the president in B's utterance is interpreted. The presupposition introduced by the definite NP the president that its referent is already established in the context thus justifies an interpretation of the nominal predicate president at the utterance time rather than at the verbal predication time in 1980.

(11) A: Gosh, the government is really pushing a hard line with these countries. B: Well, the president already made it quite clear during the incident in 1980 that he wasn't a soft guy.

Note that, in general, there seems to be no order imposed on the time at which a nominal predicate is interpreted and the verbal predication time. In (11) the nominal predicate is interpreted at the utterance time, i.e., at a time later than the verbal predication time, while the nominal predicate hostage in the context represented in (8) is interpreted at a time preceding the verbal predication time as well as the utterance time.

Partitive NPs which embed a definite NP are also interpreted with respect to a contextually established set of individuals. The partitive NP some of the professors in (12) is contextually justified to be interpreted at the utterance time rather than the verbal predication time in a way similar to the definite NP above.

The members of this department have many hidden qualities.

Some of the professors were rock stars in the 1970s.

Yet another type of NP whose interpretation depends on the context in a particular way are possessive NPs like my father in (13). The possessive determiner my requires the NP to be interpreted with respect to the speaker of the utterance. If, for instance, I utter (13), then the relational nominal father is understood to be interpreted at a time including the time of utterance, rather than the verbal predication time. Here, the contextual justification for the nominal predicate to be interpreted at a time distinct from the verbal predication time according to (5) is contributed by the interpretation of the possessive NP in context as well as the lexical semantics of the nominal predicate father which expresses a relation between individuals that, once established, pertains to the individuals even after death (see Tonhauser (2000) for a formalization of these issues).

(13) In 1963 my father biked through all of France by himself. 
In dynamic semantic accounts of NP-interpretation, the interpretation of the types of NPs just illustrated is context-dependent in one way or another: quantificational NPs are contextually restricted, and definite.and.possessive NPs refer back to contextually established individuals. These twecthanisms facilitate a contextual justification for a non-default temporal interpretation as specified by (5) for these types of NPs. NPs like indefinites or bare plurals, on the other hand, have discourse functions different from quantificational and definite NPs and are generally not interpreted with respect to a contextually established set of individuals. However, the following examples demonstrate that the context may also affect the temporal interpretation of these types of NPs.

Consider, for instance, NPs embedded within existential there-constructions. These NPs have received substantial attention in the linguistic literature (e.g., Milsark (1974) and the collection in Reuland \& ter Meulen (1987)) and it is commonly assumed that these NPs introduce new individuals to the context, as in (14). This accounts for the fact that definite and quantificational NPs are typically excluded from this type of construction. (There exist exceptions as discussed by, e.g., Keenan (1987) or Lumsden (1988); these constructions seem to have a different discourse function altogether.)

When I arrived at the party, there was an/*the angry neighbor standing on the porch.

Generally speaking, indefinite NP refer to individuals which have not yet been established in the discourse. However, these NPs, too, may be subject to contextual restrictions as demonstrated in the example in (15). There, the discourse context establishes a contextual restriction to individuals who survived the Titanic disaster, i.e., to individuals who had some function aboard the ship. The NP some crew members is interpreted with respect to this contextual restriction: via the nominal predicate crew member the NP identifies those individuals within the universe of discourse to whom the property 'crew member' applies. Thus, the nominal predicate crew member in (14) is not interpreted at the time of utterance (the verbal predication time) but rather at a contextually established time.

Context: at a reunion of the survivors of the Titanic disaster.

Look, there are even some crew members here.

Thus, while NPs introducing new discourse participants tend to introduce them with a property which is true at the verbal predication time, as this is the time most salient to the utterance, it is possible to introduce such individuals with a property salient in the context, too.

Generally, bare plurals also don't refer back to individuals already established in the context. I follow Carlson (1977) in assuming that it is the verbal predicate which determines whether a bare plural receives a generic interpretation or an existential interpretation as illustrated in (16a) and (16b), respectively.
a. Dogs bark.
b. Dogs are barking. 
Individual-level predicates like bark in (16a) trigger a generic interpretation and stage-level predicates like are barking in (16b) trigger an existential interpretation of the bare plural dogs. I claim that with bare plurals the effect of the verbal predicate on their interpretation also has consequences for the temporal interpretation of the bare plural. With individual-level predicates, bare plurals are generically interpreted; thus, the two properties expressed by the nominal and the verbal predicate, respectively, are understood to be typically true of the individuals denoted by the bare plural. It is not necessarily the case, however, that the two properties are true of an individual at the same time. (17) illustrates a generic bare plural in which the property 'nasty professor' is understood to be true of an individual at a later time than the property 'brilliant student'.

Nasty professors were brilliant students.

In (17), the past tense predication were brilliant students not only triggers the generic interpretation of the bare plural nasty professors but also justifies an interpretation of the bare plural at a time later than the verbal predication time. This seems to be possible because the past tense predication implies that the property 'brilliant student' was true at a time earlier than the time at which the property expressed by the generic bare plural nasty professors is true.

The matter is different for existential bare plurals since the verbal predicate expresses a property which is understood to be true at a particular time of a particular set of individuals rather than of 'typical' individuals as is the case with generic bare plurals. Thus, by (5), the nominal predicate of existential bare plurals tends to be interpreted at the verbal predication time. This is illustrated in (18) for the existential bare plural policemen.

When the concert started getting crazy, policemen came running into the room.

However, it is possible for the context to justify that the nominal predicate of existential bare plurals is interpreted at a time distinct from the verbal predication time. Recall the context from (15) above in which we are at a reunion of the survivors of the Titanic disaster. I might utter (19) with the existential bare plural crew members, thus referring to individuals who were formerly crew members.

Look, crew members are here, too!

Thus, even with existential bare plurals the context may affect the temporal interpretation of the nominal predicate. Note that while with the quantificational NP in (2) the lexical semantics of the properties (and world knowledge) involved were able to at least facilitate a non-default temporal interpretation and with generic bare plurals as in (18) triggered such an interpretation, this is not possible for existential bare plurals as demonstrated in (20) in which the existential bare plural is interpreted at the verbal predication time (although, again, an appropriate context might make an alternative interpretation available).

Hostages were walking around at the party. 
This again supports the claim expressed above that with NPs which refer to a particular set of individuals the lexical semantic properties of nominal and verbal predicates do not themselves fully justify a non-default temporal interpretation according to (5). With generic bare plurals, however, which in virtue of the individual-level verbal predicate receive their particular interpretation, the lexical semantic constraints may justify a non-default interpretation.

\subsection{Summary}

Concluding, I have demonstrated that it is in principle possible for the context to justify for all types of NPs a temporal interpretation of the embedded nominal predicate at a time distinct from the verbal predication time. The condition in (5) on the temporal interpretation of nominal predicates correctly accounts for the data and furthermore interacts with a general dynamic semantic account of NP-interpretation in such a way that it predicts that particular types of NPs facilitate a non-default interpretation more than others. In the next section I extend the account to include NPs in which the nominal predicate is modified by a temporal $\mathrm{N}^{\prime}$-modifier.

\section{Temporal $\mathbf{N}^{\prime}$-modifiers}

In this section I extend the account of the temporal interpretation of NPs to temporal N'-modifiers such as former, present or in the 1980s. These modifiers form part of the NP and require a temporal interpretation themselves. ${ }^{4}$ The effect common to these modifiers is that the temporal interpretation of the modified nominal predicate is resolved by the modifier. Consider the example in (21) where the temporal modifier present requires the nominal predicate mothers to be interpreted at the time of utterance. (21) thus expresses that the individuals are mothers at the utterance time and that they watched the wedding at a time prior to the utterance time (when they might not have been mothers yet).

(21) Many present mothers watched the wedding of Prince Charles and Lady Di.

Intuitively, in the light of the discussion in section 2.2 , such a temporal modifier is necessary in order to identify the desired set of individuals whenever the context alone is not sufficiently restrictive. For (21) this means that since the context is not restricted to individuals who are mothers at the utterance time, this is made explicit with the temporal modifier present.

In the compositional analysis of present mothers in (22) the modifier present introduces a time DR $\mathrm{t}^{\prime}$ which is identified with the utterance time $\mathrm{n}$. This temporal DR $t^{\prime}$ is required to be identified with the the temporal DR of the element modified. Hence, the temporal DR introduced by mother is identified with $n$ via $\mathrm{t}^{\prime} .{ }^{5}$ 
(22)

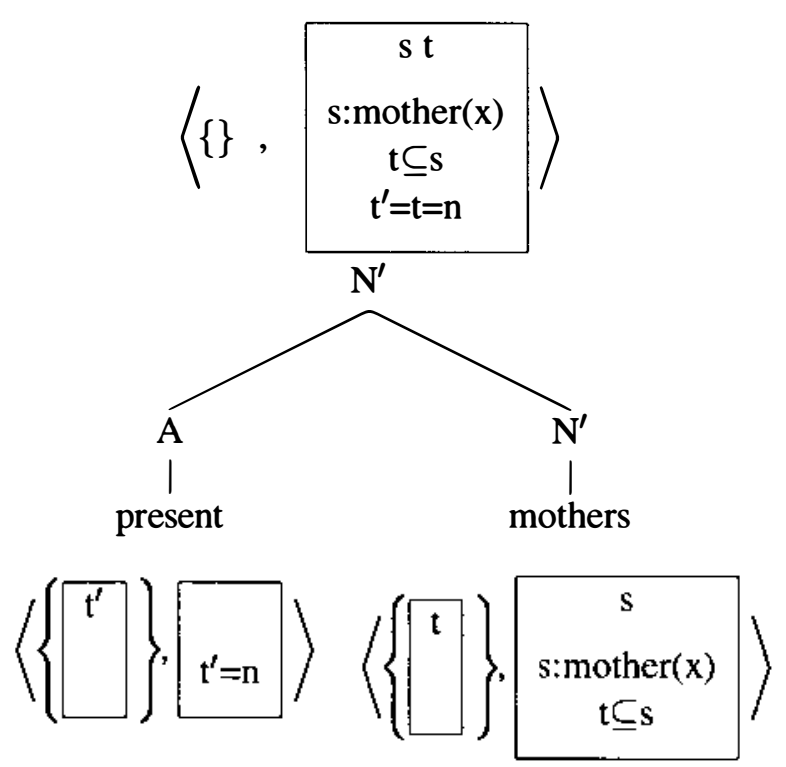

The temporal DR t introduced by the nominal predicate mother in (21) is already explicitly bound during its composition with present and need not be resolved according to (5) anymore. The resolved DRS of (21) in (23) specifies that the individuals $\mathrm{x}$ are mothers at the time of utterance and that they were watching the wedding at some time in the past; crucially, the temporal DR $t$ of the nominal predicate is not resolved to $t_{t}$ but identified with $\mathrm{n}$ according to (22).

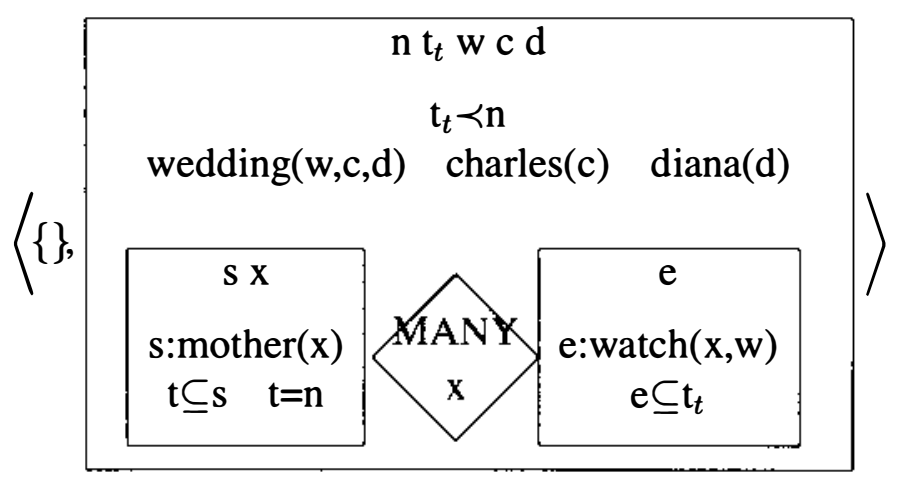

Without the contribution of the temporal modifier present, the nominal predicate mother in (24) is interpreted by the default in (5) at the verbal predication time.

$$
\text { Many mothers watched the wedding of Prince Charles and Lady Di. }
$$

The uninterpretability of (25) is accounted for if we assume that both temporal $\mathrm{N}^{\prime}$ modifiers in (25) introduce a temporal DR which is required to bind the temporal DR of the modified element. Since the time introduced by present does not overlap with the time introduced by the 1980s it is clear that the nominal predicate mothers cannot receive its temporal location from both modifiers.

\#Many present mothers of the 1980s watched the wedding of Prince Charles and Lady Di. 
Another interesting set of temporal modifiers are former and future. They differ from temporal $\mathrm{N}^{\prime}$-modifiers such as present in that they do not require the modified element to be interpreted at a particular time, but rather make available a post- or pre-state, respectively, of the property expressed by the modified element. For instance, (26) specifies that many individuals are working in some pizzeria at the time of utterance and that these individuals are former students at the time of utterance rather than actual students. Thus, former introduces a state which follows the state introduced by the nominal predicate student.

Many former students are working in this pizzeria.

In the compositional account of former students in (27) I propose that former, parallel to nominal predicates, introduces a state DR $s^{\prime}$. This state DR represents the time during which the property expressed by the element modified does not hold anymore (since intuitively, e.g., a former student is not a student anymore) and thus $s^{\prime}$ immediately follows the state $s$ introduced by the modified element, as indicated by the condition $s \propto s^{\prime}$. The temporal DR $t$ of the nominal predicate is resolved in the composition. The DR $s^{\prime}$ itself is temporally located by a temporal DR $t^{\prime}$ which must be contextually resolved (see below).

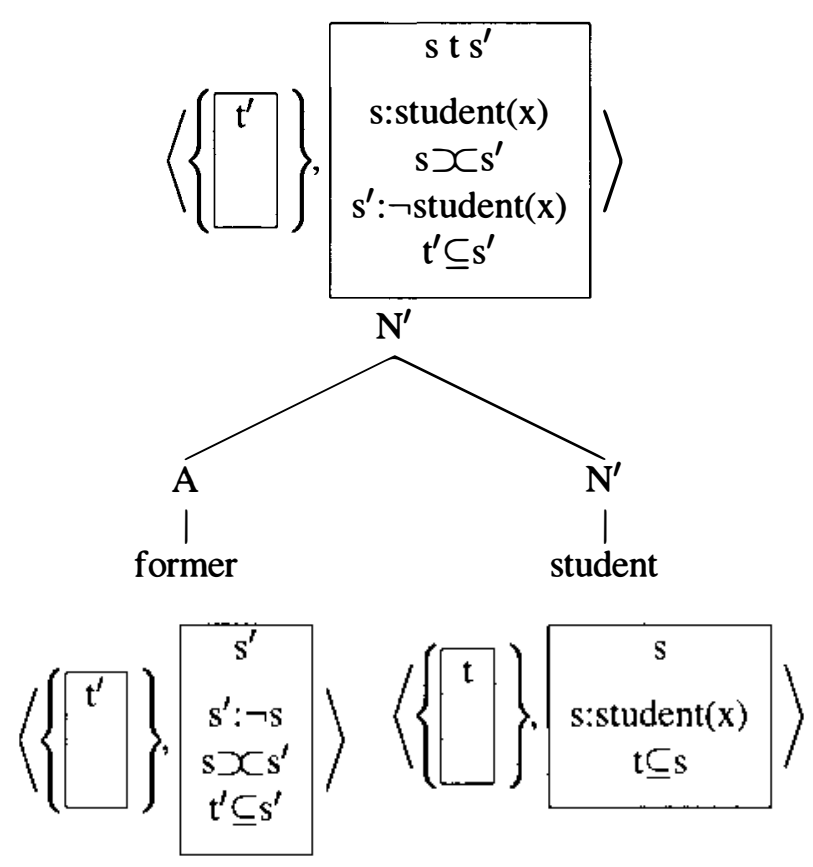

As mentioned above, we understand (26) to express that the individuals are former students at the time at which they are working in the pizzeria. Thus, the temporal DR $t^{\prime}$ introduced by the modifier former is resolved to the verbal predication time. I assume that the resolution conditions given in (5) for nominal predicates apply also to other elements within the $\mathrm{N}^{\prime}$ that express properties or relations which need to be temporally interpreted. ${ }^{6}$ Thus, by (5), the temporal DR $\mathrm{t}^{\prime}$ introduced by former is resolved to the verbal predication time as given in (28). Note that nothing in (28) requires that these individuals are students at a particular time; in fact, they might 
have been students at rather distinct times in the past of the utterance time.

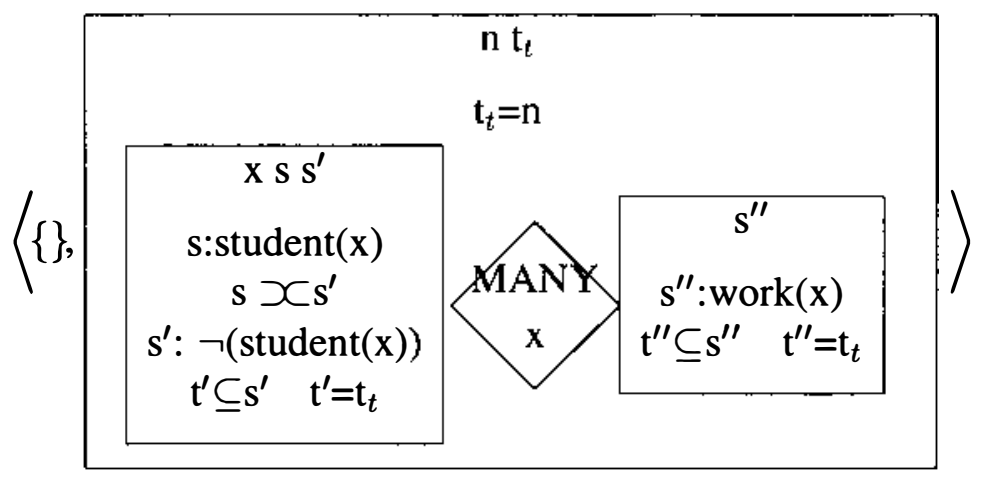

A third set of modifiers in the examples in (29) point to another important aspect of the temporal interpretation of NPs, namely that the temporal interpretation of NPs may also influence the temporal interpretation of the verbal predicate. The prepositional temporal $\mathrm{N}^{\prime}$-modifiers in (29) all have in common that they additionally introduce a time at which the nominal predicate is interpreted. However, they differ with respect to the constraints they impose on the temporal interpretation of the verbal predicate. I refer to the type of modifier in (29a) as a precedence modifier, to the one in (29b) as an inclusive, and to the one in (29c) as a neutral modifier. These classifications are based on the semantic constraints the modifiers impose on the temporal interpretation of the verbal predicate: ${ }^{7}$ the precedence modifier in (29a) requires that the time introduced by the prepositional modifier precedes the verbal predication time, while the inclusive modifier in (29b) requires that the time introduced by the modifier subsumes the verbal predication time. The neutral modifier in (29c) does not introduce constraints on the verbal predication time. ${ }^{8}$
a. Singers from the eighties are no longer popular.
b. Most women in the eighties did not want to join the army.
c. 2001 was the year when pop stars of the eighties reclaimed their thrones.

These modifiers, like present, require the modified nominal predicate to be interpreted at the time specified by the modifier. Precedence and inclusive modifiers differ from from present in that they introduce constraints on the verbal predication time. In the examples in (30a) and (30b) the constraints introduced by precedence and inclusive modifiers, respectively, are violated.

(30) a. \#Most students from the eighties were aware of this album in 1980.

b. \#Women soldiers in the eighties are not part of the front line troops today.

Similarly to the analysis of present, the temporal NP embedded within these prepositional modifiers introduces a time $t$, which resolves the temporal DR of the modified nominal. However, in order to account for the constraints on the verbal predication time $t_{t}$, these modifiers also introduce a constraint on the temporal relation of the time $t$ with the verbal predication time $t_{t}$, as illustrated in (31): in the case of precedence modifiers (a), the preposition requires the time $t$ introduced by the tem- 
poral NP to precede the verbal predication time; an inclusive modifier (b) requires the temporal NP to locate the verbal predication time.

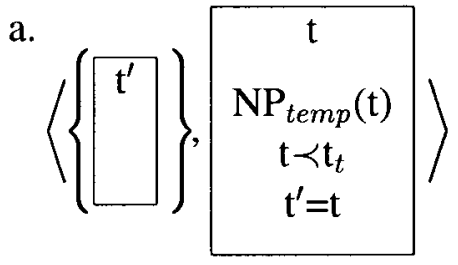

b.

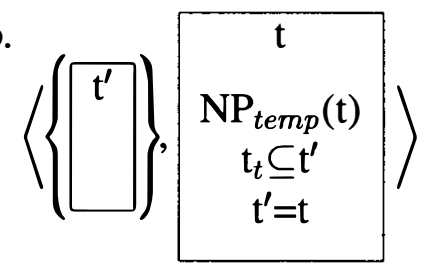

Temporal $\mathrm{N}^{\prime}$-modifiers parallel adverbial temporal modifiers of verbal projections to the extent that they require the modified element to be interpreted at the time introduced by the temporal modifier. This furthermore supports the assumption made in this paper that the representation of nominal predicates is identical to that of verbal predicates assumed, e.g., in Kamp \& Reyle (1993).

Concluding, I have argued in this and the preceding section that the temporal interpretation of an NP is contextually determined: a nominal predicate is temporally interpreted at the verbal predication time unless there is contextual justification to the contrary. In section 2 I illustrated a number of ways in which the interpretation of NPs in context can justify a non-default temporal interpretation of the nominal predicate. In this section, I have extended the contextual justification to temporal $\mathrm{N}^{\prime}$-modifiers which may provide for a time at which the modified nominal predicate is interpreted. I have also argued that the condition in (5) should be extended to include other elements within the $\mathrm{N}^{\prime}$-projection, like former, as in (32).

CONDITION ON THE TEMPORAL INTERPRETATION OF AN NP

The $\mathrm{N}^{\prime}$-projection of an NP is temporally interpreted at the verbal predication time, unless there exists contextual justification to the contrary.

It has become apparent from the discussion of prepositional modifiers that the temporal interpretation of NPs may influence the temporal interpretation of the verbal predicate; thus, the interaction between the temporal interpretation of nominal and verbal predicates and their respective projections should be regarded as bidirectional. In the next section I discuss the account presented in Musan $(1995,1999)$ which claims that there exists a particular set of NPs, which must be interpreted at the verbal predication time.

\section{Musan's Temporally (In)Dependent NPs}

Renate Musan develops an account of the temporal interpretation of NPs (Musan $(1995,1999))$ in which the determiner of the NP plays a central role in whether an NP is interpreted at the verbal predication time or not. Musan argues that there exist two types of NPs: presuppositional NPs, which are free to be interpreted at any time available in the context, and cardinal NPs, which must be interpreted at the verbal predication time. ${ }^{9}$ Following Milsark (1974), Musan assumes that strong determiners like every, each and most form presuppositional NPs, while weak determiners like many, some and few can form either presuppositional or cardinal NPs. The position of the NP and stress determines whether a weak determiner forms a 
presuppositional or a cardinal NP (Musan (1995:143)):

Presuppositional NPs are (i) partitive DPs, (ii) DPs with strong determiners, (iii) DPs with weak determiners that are stressed on the determiner, (iv) and generic bare plural noun phrases.

Cardinal NPs are (i) DPs with weak determiners in certain syntactic positions, (ii) existential bare plurals and (iii) DPs with weak determiners that are stressed on the noun.

In Musan's account, the $\mathrm{N}^{\prime}$-material of a presuppositional NP is mapped to the restriction of the determiner and may thus be interpreted at a time distinct from the verbal predication time. Cardinal NPs, however, interpret the $\mathrm{N}^{\prime}$-material as well as the VP-material within the scope of the determiner and thus require the $\mathrm{N}^{\prime}$ and the VP to be interpreted at the same time. Crucially, Musan assumes that cardinal NPs do not quantify over individuals in their whole temporal extendedness but over 'stages', i.e., spatio-temporal parts of individuals. The temporal location and extension of the stage is determined by tense, temporal adverbials and contextual factors. Thus, nominal predicates in cardinal NPs do not introduce a temporal argument but receive their temporal interpretation indirectly via the individual variable which is restricted to a particular time.

Musan's claim that cardinal NPs form a class of NPs which must be temporally interpreted at the verbal predication time can be empirically invalidated. Thus, while I agree with Musan that the determiner of an NP influences the temporal interpretation of the $\mathrm{N}^{\prime}$-projection (as demonstrated in section 2.2), there exist contexts in which Musan's cardinal NPs may receive a temporal interpretation at a time distinct from the verbal predication time, contrary to Musan's claim. I illustrate this for each type of Musan's cardinal NPs in (33). For the type of NPs in (33i), Musan gives two syntactic positions, namely inside the VP (in German) and in the context of existential there-constructions. However, Musan (1995:58) notes that the interpretation in the former environment is only a preference. For the latter, i.e., existential there-constructions, I have presented in (15) above an NP within an existential sentence which is temporally interpreted at a time distinct from the verbal predication time. The second group in (33), existential bare plurals, have been discussed in section 2, too, and the example in (19) presents a counterexample to Musan's claim that these NPs must be interpreted at the verbal predication time. Clearly, all these NPs show a tendency to be interpreted at the verbal predication time because their interpretation does not depend on the context as much as, for instance, that of definite or quantificational NPs. This, however, does not categorically rule out the possibility that the nominal predicate is temporally interpreted at a time distinct from the verbal predication time. Finally, Musan's third type of cardinal NPs in (33iii), i.e., 'DPs with weak determiners that are stressed on the noun', is refined in Musan (1999:629) to 'DPs with weak determiners that have a rising accent on the noun'. A rising accent on a nominal predicate can indicate contrastive focus and thus does not guarantee that the NP is interpreted at the verbal predication time. Consider the NP some brides in (34) which receives a rising 
accent on the noun and should thus be a cardinal NP according to Musan. However, in the given context, the rising stress is interpreted contrastively and the nominal predicate is interpreted at the utterance time rather than the verbal predication time. Thus, again, contrary to Musan's claim, the cardinal NP in (34) is not interpreted at the verbal predication time.

(34) Context: at a mass wedding, after the priest has joined the 500 couples.

I bet 10 years ago most of the grooms weren't even thinking of getting married while some BRIdes were already getting measured for the dress.

I have presented counterexamples for each of the three types of Musan's cardinal NPs as given in (33(i)-(iii)). Therefore, I conclude that there exists no empirical evidence for Musan's class of NPs which are obligatorily interpreted at the verbal predication time. Furthermore, Musan's distinction between cardinal and presuppositional NPs based on Milsark (1974) is not independently motivated theoretically either: Milsark proposes a characterization of NPs according to their determiner to account for their distribution in existential there-constructions. That this is not sufficient has been pointed out by a number of people (e.g., Lumsden (1988)). Finally, Musan's account does not determine the temporal interpretation of a large set of NPs, namely the presuppositional NPs which she claims can be temporally interpreted at any contextually available time. Musan (1995:III.8) acknowledges that the factors I discuss in section 2 may influence the temporal interpretation of presuppositional NPs, but she claims that they are still in principle temporally independent. Note that in my account these factors are central, and, furthermore, that the dynamic semantic analysis I developed above assigns a particular temporal interpretation to all occurrences of NPs. In the next section I briefly discuss some cross-linguistic evidence which provides further support for the dynamic semantic analysis I developed in sections 2 and 3.

\section{Cross-linguistic Considerations}

I believe that cross-linguistic data provides further support for a dynamic account in which nominal and verbal predicates are treated in parallel: Nordlinger \& Sadler (2001, ms.) present a typological survey of languages in which nominal predicates are inflected with tense. Some of these languages have paradigms for verbal tense, too, and some only have tense inflection on nominal predicates. An example of the former type is Tariana, an Arawak language from north-west Amazonia, Brazil. Nominal predicates can be inflected for past or future tense, which affects only the temporal interpretation of the nominal predicate; a separate tense system is available for verbal predicates. Nordlinger \& Sadler (2001, ms.) report (following Aikhenvald (ms.)) that about $40 \%$ of nominal predicates in natural discourse are inflected for tense. The future tense marker - pena of nominal predicates is illustrated in wa-t fimari-pena (1PL-son.in.law-NOM.FUT) 'our future son-in-law' and pi-ya-dapana-pena (2SG-POSS-house-NOM.FUT) 'your future house'. The nominal past tense marker has three forms (fem/masc singular distinction and a plural form) and is illustrated in correio-miki-ri (post-office-PST-NF) 'old/former 
post office' and $d u$-sa-do-mik-ru (3SG.NF-spouse-FEM-NOM.PST-FEM) 'his late spouse'. Nordlinger \& Sadler (2001) report that verbal tense in Tariana is a floating clitic which attaches to any focussed constituent in a clause, including nominals, in which case both nominal and verbal tense can be marked on a nominal. Nordlinger \& Sadler (2001, ms.) present many more languages in which nominal predicates are inflected with tense morphology. It is clear that a closer look at these languages is needed to establish the possibilities with respect to the morphosyntactic realization of tense and its interpretation; however, the fact that languages exist which treat nominal and verbal predicates in a parallel manner with respect to temporal interpretation provides additional motivation for the representation of nominal predicates I proposed in section 2. This representation allows to account for nominal and verbal tense inflection in a parallel fashion. An account like Musan's in which nominal predicates do not receive a temporal argument of their own, but in which temporal interpretation is fixed by stage-level quantification cannot easily account for such languages.

\section{Conclusions}

I have argued in this paper that the temporal interpretation of nominal predicates (and their $\mathrm{N}^{\prime}$-projections) is contextually determined: a nominal predicate is interpreted at the verbal predication time unless there exists contextual justification to the contrary. This contextual justification includes temporal $\mathrm{N}^{\prime}$-modifiers which specify the time at which the nominal predicate modified is temporally interpreted. The cross-linguistic data as well as the discussion of temporal $\mathrm{N}^{\prime}$-modifiers motivate a parallel treatment of the temporal interpretation of nominal and verbal predicates, which is captured in the analysis developed here. Partee $(1973,1984)$ has demonstrated a number of parallels between the resolution of nominal and verbal anaphora. The dynamic semantic account of the temporal interpretation of NPs developed here can thus be argued to extend Partee's observations to the temporal domain. Furthermore, the analysis can contribute to a general account of the temporal interpretation of natural language expressions. Future analyses of cross-linguistic data could point to further generalizations about the morpho-syntactic realization and interpretation of tense.

\section{Endnotes}

* This study presents revised material from my MA thesis (Tonhauser (2000)); I thank Hans Kamp for invaluable comments, as well as Ash Asudeh, David Beaver, John Beavers, Lev Blumenfeld, Brady Clark, Hana Filip, Iván García, Florian Jaeger, Beth Levin, Ivan Sag, Arnold Zwicky and the audience of SALT 12 at San Diego. At Stuttgart, this research was supported by the Sonderforschungsbereich (SFB) 340 Linguistic Foundations for Computational Linguistics which I gratefully acknowledge. The usual disclaimers apply.

${ }^{1}$ Contrary to Enç's (1986: 420) claim that only nominal and verbal predicates need to receive a temporal interpretation, I claim that (at least) possessives and adjectives also need to introduce a temporal argument since they express properties pertaining 
to individuals at particular times.

2 There exist prefixes Alt- (for German) and $e x$ - (for English and German) which indicate that the property expressed by the nominal predicate modified is true at a prior time, but these prefixes are not productive.

3 This argumentation also accounts for another of Enş's (1981) famous examples as given in (i) where lexical semantic constraints seem to license a non-default temporal interpretation but, in fact, contextual justification is needed, too.

(i) The fugitives are in jail now.

Enç (1981)

${ }^{4}$ Clearly, non-temporal modifiers like beautiful or daring also need to receive a temporal argument to express the time at which the property expressed by the modifier is true of the individuals. I assume here that these properties are interpreted by a condition similar to (5).

${ }^{5}$ Note that current, unlike present, seems not to require an interpretation at the time of utterance but allows an anaphoric interpretation at the verbal predication time.

(ii) In 1985 Ronald Reagan remarked that current actors had less potential to become president of the USA.

6 Since the resolution of the temporal DR introduced by former is guided by a condition similar to the one given in (5), this allows to account for the following real example, which I owe to Ash Asudeh. The day after the 2002 California elections, a radio announcer referred to the defeated candidate with the NP the former secretary of state as in (iii) although the individual was still secretary of state at the utterance time.

(iii) The former secretary of state attempted to run on her name alone.

${ }^{7}$ Parallel modifiers exist in German; see Tonhauser (2000).

${ }^{8}$ Musan (1995:142) claims that precedence modifiers do not interact with the verbal predication time.

${ }^{9}$ Musan $(1995,1999)$ presents some exceptions to this correlation, but they are irrelevant for the discussion here.

\section{References}

Aikhenvald, Alexandra (ms.) The Tariana language of Northwest Amazonia. Carlson, Greg (1977). Reference to Kinds in English. PhD thesis, University of Massachusetts at Amherst.

Enç, Mürvet (1981). Tense without Scope: An Analysis of Nouns as Indexicals. Ph.D. thesis, University of Wisconsin, Madison.

Enç, Mürvet (1986). Towards a Referential Analysis of Temporal Expressions. Linguistics and Philosophy, 9(4).

Kamp, Hans (1981). A Theory of Truth and Semantic Representation. In Groenendijk, J., Janssen, T., \& Stokhof, M. (eds.), Truth, Representation and Information. Foris, Dordrecht.

Kamp, Hans (2001a). The Importance of Presupposition. In Rohrer, C., \& Ross- 
deutscher, A. (eds.), Linguistic Form and Its Computation. CSLI publications, Stanford University.

Kamp, Hans (2001b). Presupposition Computation and Presupposition Justification. In Bras, M. \& Vieu, L. (eds.), Semantics and Pragmatics of Discourse and Dialogue: experimenting with current theories. Elsevier.

Kamp, Hans \& Reyle, Uwe (1993). From Discourse to Logic. Kluwer, Dordrecht.

Keenan, Ed (1987). A Semantic Definition of 'Indefinite NP'. In The Representation of (In)definiteness. Reuland, E. \& ter Meulen, A. (eds.), MIT Press, Cambridge, Mass.

Lumsden, Michael (1988). Existential Sentences. Their Structure and Meaning. Croom Helm, New York.

Milsark, Gary L. (1974). Existential Sentences in English. Ph.D. thesis, MIT.

Musan, Renate (1995) On the Temporal Interpretation of Noun Phrases. PhD thesis, MIT. Published 1997 by Garland, New York.

Musan, Renate (1999). Temporal Interpretation and Information-Status of Noun Phrases. Linguistics and Philosophy, 22(6).

Nordlinger, Rachel \& Sadler, Louisa (2001). Nominal tense with nominal scope: a preliminary sketch, Proceedings of the LFG01 Conference, CSLI publications, Stanford, CA.

Nordlinger, Rachel \& Sadler, Louisa (ms.). 'There is your house-PAST': a typological survey of nominal TAM inflection. February 2002.

Partee, Barbara (1973). Some structural analogies between Tenses and Pronouns in English. Journal of Philosophy, 70.

Partee, Barbara (1984). Nominal and Temporal Anaphora. Linguistics and Philosophy, 7.

Reyle, Uwe, Rossdeutscher, Antje \& Kamp, Hans (2000). Ups and downs in the theory of temporal reference. In Reyle, U. (ed.) Presuppositions and Underspecification in the Computation of Temporal and other Relations in Discourse. Working paper no.164 SFB 340, Stuttgart/Tübingen.

Reuland, Eric \& ter Meulen, Alice (1987). The Representation of (In)definiteness. (eds.) MIT Press, Cambridge, Mass.

Tonhauser, Judith (2000) Dynamic Semantics and the Temporal Interpretation of Noun Phrases. Diploma Thesis, University of Stuttgart.

van der Sandt, Robert (1992). Presupposition Projection as Anaphora Resolution. Journal of Semantics, 9(4). 